\title{
Disrupted epithelial barrier in nasal polyps characterizes aspirin exacerbated respiratory disease
}

Anand Kumar Andiappan ${ }^{1}$, Mohammad Asad ${ }^{2}$, Celine Chua ${ }^{3}$, Esha Sehanobish ${ }^{2}$, Zhen Ren $^{4}$, Xue Ying Chan ${ }^{1}$, Josephine Lum ${ }^{1}$, Nicholas Ang ${ }^{1}$, Kaibo Duan ${ }^{1}$, Adam Gersten ${ }^{2}$, Waleed M. Abuzeid ${ }^{5}$, Nadeem Akbar ${ }^{2}$, Marc Gibber ${ }^{2}$, Shanshan Howland ${ }^{1}$, Bernett Lee ${ }^{1}$, Olaf Rotzschke ${ }^{1}$, Steven A. Porcelli ${ }^{2}$, and Elina Jerschow ${ }^{2}$

${ }^{1}$ Singapore Immunology Network

${ }^{2}$ Montefiore Medical Center

${ }^{3}$ National University of Singapore Department of Biological Sciences

${ }^{4}$ Washington University in St Louis John T Milliken Department of Medicine

${ }^{5}$ University of Washington Department of Otolaryngology Head and Neck Surgery

September 25, 2021

Disrupted epithelial barrier in nasal polyps characterizes aspirin exacerbated respiratory disease

Anand Kumar Andiappan ${ }^{*}$, Mohammad $\mathrm{Asad}^{2}$, Celine Chua ${ }^{3}$, Esha Sehanobish ${ }^{2}$, Zhen Ren ${ }^{4}$, Xue Ying Chan $^{1}$, Josephine Lum ${ }^{1}$, Nicholas Ang ${ }^{1}$, Duan Kaibo ${ }^{1}$, Adam Gersten ${ }^{2}$, Waleed M. Abuzeid ${ }^{5}$, Nadeem Akbar $^{2}$, Marc Gibber ${ }^{2}$, Shanshan Howland ${ }^{1}$, Bernett Lee ${ }^{1}$, Olaf Rotzschke ${ }^{1}$, Steven A. Porcelli ${ }^{2}$, Elina Jerschow $^{2 *}$

1 Singapore Immunology Network, Agency for Science, Technology and Research

2 Albert Einstein College of Medicine/Montefiore Medical Center, Bronx, NY, USA

${ }^{3}$ Department of Biological Science, National University of Singapore

${ }^{4}$ Division of Allergy and Immunology, Department of Medicine, Washington University Schoolf of Medicine, St Louis, MO, USA

${ }^{5}$ Rhinology and Endoscopic Skull Base Surgery, Department of Otolaryngology - Head and Neck Surgery, University of Washington, Seattle, WA, USA

*Correspondence: EJERSCHO@montefiore.org; anand_andiappan@immunol.a-star.edu.sg

\section{Conflict of interest:}

:

For Elina Jerschow: Advisory Board - GlaxoSmithKline, Sanofi-Regeneron, Novartis/Genentech.Consultant -GlaxoSmithKline, none of these are relevant to this work.

Other authors declare no conflict of interest

\section{Funding:}

This work was supported by CTSA grant number 5KL2TR001071from the National Center for Advancing Translational Sciences (NCATS), a component of the National Institutes of Health (NIH). Portions of this 
work were funded by R21AI146804 and the Stony Wold-Herbert Fund research grant (to EJ). Its contents are solely the responsibility of the authors and do not represent the official views of the NIH. A.K. Andiappan was supported by an NMRC (National Medical Research Council) YIRG (Young Individual Research Grant) grant (OFYIRG17nov065), an Agency for Science, Technology and Research CDA (Career Development Award) grant (202D800012),

Keywords : asthma, chronic rhinosinusitis, nasal polyps, barrier dysfunction, AERD

\section{To the Editor}

Chronic rhinosinusitis with nasal polyps (CRSwNP) is a debilitating inflammatory disease of the sinonasal cavities. ${ }^{1}$ Concomitant CRSwNP and asthma, together with hypersensitivity reactions to cyclooxygenase-1 (COX-1) inhibitors, is a particular phenotype known as aspirin exacerbated respiratory disease (AERD), which is associated with greater disease severity. ${ }^{2}$

In this study, we attempted to characterize clinical, laboratory, and transcriptomic differences between patients with AERD ( $\mathrm{N}=13)$ and $\mathrm{CRSwNP}$ patients without AERD (N=13).

We compared patient demographics and clinical characteristics (Table 1 ). A more severe clinical phenotype with asthma presence and a higher number of sinus surgeries was noticed in AERD patients. LM scores in AERD were significantly higher compared to CRSwNP patients (20 (IQR 16-23) vs. 14 (IQR 14-18), respectively, $\mathrm{P}=0.0065$, Figure $\mathbf{1 A}$ ). Peripheral blood eosinophil counts were not significantly different between the groups (Figure E1A ). However, blood eosinophil counts had a positive correlation with LM scores in CRSwNP patients $(\mathrm{R}=0.65, \mathrm{P}=0.018$, Figure E1C $)$, while this correlation was absent in AERD $(\mathrm{R}=-0.33, \mathrm{P}=0.26$,Figure E1B) . Neutrophil counts were higher in the polyp tissues of AERD patients (8.5 cells per hpf (IQR 3-25) vs. 2.5 cells (IQR 2-5) in CRSwNP (P =0.026, Figure 1B ). Analysis of the inflammatory cell infiltrates on histologic slides showed that both groups had abundant but similar eosinophil counts (Figure 1C).

We then conducted the whole-transcriptomic sequencing, followed by pairwise comparisons of gene expression between the AERD and CRSwNP polyps. A stringent analysis of the transcriptomic data using a false discovery correction (FDR) narrowed down the differentially expressed genes (DEGs) to 10 protein-coding genes significantly downregulated in AERD compared to CRSwNP (Figure 1D, Table E1). In AERD, there was a downregulation of the genes coding for epidermal differentiation marker (CALM3, CRNN), keratinsation (CNFN ,KRT13 , SPINK5 ), secreted mucin protein MUC2,and epidermal barrier marker genes (SPINK5, CRNN, A2ML1). Functional analysis of significant DEGs using Ingenuity Pathway Analysis highlighted biological pathways enriched with AERD status such as organismal injury $(\mathrm{N}=264, \mathrm{P}=1.66$ x $\left.10^{-6}\right)$, inflammatory responses $\left(\mathrm{N}=147, \mathrm{p}=5.95 \times 10^{-20}\right)$, humoral immune response $(\mathrm{N}=34, \mathrm{p}=5.95 \mathrm{x}$ $\left.10^{-20}\right)$, and cell morphology $\left(\mathrm{N}=73, \mathrm{p}=4.45 \times 10^{-14} ;\right.$ Figure 1E-G $)$.

Taken together, these findings underline the key differences in patients with CRSwNP, with and without AERD. Compared to CRSwNP patients, we observed that AERD patients were likely to have a more severe clinical disease. This was associated with a downregulation of genes responsible for the epithelial barrier function. AERD has a strong type- 2 inflammation, but a simultaneous increase in the presence of neutrophils suggests that AERD is characterized by mixed Th17 along with Th1 and Th2 immune responses assocaited with eosinophils ${ }^{3}$ While blood eosinophils were not sufficiently predictive of type 2 inflammation in patients with chronic rhinosinusitis without nasal polyps, ${ }^{4}$ here we show that peripheral blood eosinophilia was significantly associated with LM scores in CRSwNP patients. This finding was not relevant to AERD patients, indicating that other cells, perhaps neutrophils, contribute to the severity of the polyp burden in AERD. The presence of a mixed eosinophilic and neutrophilic inflammation has recently been reported in a more severe phenotype of CRSwNP. ${ }^{5}$ Such phenotype is likely due to the presence of Charcot-Leyden crystals that attract neutrophils and lead to a greater barrier dysfunction and bacterial colonization. ${ }^{5,6}$ Our observations also suggest that the clinical characteristics of AERD, as a more severe phenotype of CRSwNP, are linked to a distinct dysregulation of epithelial barrier function in AERD polyps, offering a basis for further mechanistic studies on epithelial remodeling and inflammation of the upper and lower airways. 


\section{References}

1. Khan A, Vandeplas G, Huynh TMT, Joish VN, Mannent L, Tomassen P, et al. The Global Allergy and Asthma European Network (GALEN rhinosinusitis cohort: a large European cross-sectional study of chronic rhinosinusitis patients with and without nasal polyps. Rhinology. 2019;57(1):32-42.

2. Kim JE, Kountakis SE. The prevalence of Samter's triad in patients undergoing functional endoscopic sinus surgery. Ear Nose Throat J. 2007;86(7):396-9.

3. Celejewska-Wójcik N, Wójcik K, Ignacak-Popiel M, Ćmiel A, Tyrak K, Gielicz A et al.. Subphenotypes of nonsteroidal antiinflammatory disease-exacerbated respiratory disease identified by latent class analysis. Allergy $2020 ; 75: 831-840$.

4. Delemarre T, Holtappels G, De Ruyck N, et al. Type 2 inflammation in chronic rhinosinusitis without nasal polyps: Another relevant endotype. J Allergy Clin Immunol. 2020;146(2):337-343.e6.

5. Delemarre T, Holtappels G, De Ruyck N, Zhang N, Nauwynck H, Bachert C, et al. A substantial neutrophilic inflammation as regular part of severe type 2 chronic rhinosinusitis with nasal polyps. J Allergy Clin Immunol. 2021;147(1):179-88 e2.

6. Delemarre T, Bochner BS, Simon HU, Bachert C. Rethinking neutrophils and eosinophils in chronic rhinosinusitis. J Allergy Clin Immunol. 2021;148(2):327-35.

\section{Figure legends}

\section{Figure 1: Characteristics of the study cohort stratified by AERD status}

Lund-Mackay (LM) score. (B) Peripheral blood eosinophil counts (cells/ $\mu$ l). Correlation between peripheral blood eosinophil count (cells/ $\mu$ l) to LM score for CRSwNP (C) and AERD(D) . Count/hpf for (E ) eosinophils and $(\mathbf{F})$ neutrophils from histologic analysis of nasal polyp tissue. Ingenuity pathway analysis (IPA) of differentially expressed genes (DEGs) highlighting enriched biological pathways under (G ) Diseases and Disorders, (H ) Molecular and Cell Function and (I ) Physiological system based on a cut off of nominally significant $\mathrm{P}$-value $<0.05$.

\section{Hosted file}

Figure 1.pptx available at https://authorea.com/users/436799/articles/538872-disruptedepithelial-barrier-in-nasal-polyps-characterizes-aspirin-exacerbated-respiratory-disease

\section{Hosted file}

Table 1.docx available at https://authorea.com/users/436799/articles/538872-disruptedepithelial-barrier-in-nasal-polyps-characterizes-aspirin-exacerbated-respiratory-disease 